\title{
Introducing a HPV Vaccination Program: The Experience in the State of Geneva, Switzerland (2007-2009)
}

\author{
Emilien Jeannot $^{1 *}$, Philippe Sudre ${ }^{2}$, Philippe Chastonay ${ }^{1}$ \\ ${ }^{1}$ Institute of Social and Preventive Medicine, Faculty of Medicine, University of Geneva, Geneva; Switzerland; ${ }^{2}$ Service of Epidemiol- \\ ogy and Contagious Diseases, State Medical Office, Department of the Regional Affairs, Economy and Health, Geneva, Switzereland. \\ Email: emilien.jeannot@unige.ch
}

Received January $25^{\text {th }}, 2011$; revised February $24^{\text {th }}, 2011$; accepted February $25^{\text {th }}, 2011$.

\begin{abstract}
Objective: The objective of this study is to assess the HPV vaccination coverage of 11 to 19 year-old girls during a state coordinated HPV vaccination program in Geneva, Switzerland, from September 2007 to December 2009. Methods: The $H P V$ vaccination program was coordinated by the State Medical Office. It relied on practitioners in private practice, on the School Health Service and on a temporary vaccination structure at the Geneva University Hospital. Each service provider transmitted quarterly to the State Medical Office the nominal list of the persons who had received their first, second or third shot. The data were processed through the Téléform (c) software and analyzed to assess the HPV coverage of the target population. Results: The global coverage rates on December $31^{\text {st }} 2009$, 2 years after the program had been launched, were $48.1 \%$ for one dose, $43.7 \%$ for two doses and $41.6 \%$ for three doses of the HPV vaccine. These rates were quite different from one cohort to another: for girls born in 1995 (14 year-old), cover rates were 70\% for the first, $68.1 \%$ for second and $65.1 \%$ for the third dose. Conclusion: This study shows that it is possible to obtain a high coverage rates after two years of a state coordinated HPV vaccination program.
\end{abstract}

Keywords: Immunization, Adolescent, Human Papilloma Virus

\section{Introduction}

The human papilloma virus (HPV) is the most frequent cause of sexually transmitted infections in Switzerland [1]. HPV is responsible, exclusively or not, for a large number of pathologies: cervical carcinoma in situ or invasive ( $100 \%$ of the cases); cancers of the anus $(90 \%)$; cancers of the penis (40\%); cancers of the vulva and the vagina (40\%); cancers of the mouth and the oro-pharynx (3\% - 12\%); genital condylomas. There are more than 120 genotypes of HPV. HPV 16 and 18 are directly responsible for the majority of the cervical cancers, HPV 6 and 11 for the condylomas. These viruses are widespread and the infection, which generally goes unnoticed, can be transmitted during the first sexual intercourse. Cancers may develop 15 to 20 years after infection [2-4].

Cervical cancer is a major public health problem. It affects approximately 1.4 million persons worldwide, with an annual incidence of 500000 cases [5,6]. Worldwide, it is responsible for 274000 deaths a year. In Europe cervical cancer affects approximately 60000 women and is responsible for 30000 deaths every year [7].

In Switzerland, every year 5000 young women present a precancerous lesion of the cervix or an in situ carcinoma, and 320 an invasive cervical carcinoma requiring a surgical/laser treatment, half of the women present a precancerous lesion of the cervix or an in situ carcinoma are less than 50 years old when the disease is diagnosed. Cervical cancer is the second cause of cancer in women in Switzerland [8].

In Geneva, there are approximately 400 cases of in situ and 30 invasive cervix cancers every year, resulting in 5 to 10 deaths per year [9].

The first vaccine to prevent cervical cancer and precancerous genital lesions caused by human papilloma virus, (Gardasil ${ }^{\circledR}$ ), was approved by the Swiss $>$ Drug Regulation Agency in 2006. To be completely effective, three vaccine doses are necessary within six months and should be administered before the beginning of sexual activity. 
In terms of cancer prevention a large proportion of persons in a community should be vaccinated, first in order to offer them personal protection and second to stop the inter-human transmission of the virus, though the benefit will appear with a delay of a decade or more [10].

The aim of this study was to assess the HPV coverage among 11 to 19 year old girls, from September 2007 to December 2009 in the Swiss canton of Geneva.

\section{Methods}

The HPV Vaccination Program in Geneva: The Swiss Federal Vaccination Committee recommended HPV vaccination free of charged for girls, aged 11 to 19 [8], if in the context of a "state vaccination program" [3].

The Geneva HPV vaccination program was coordinated by the State Medical Office. It relied on practitioners in private practice (especially pediatricians, gynecologists, internists and general practitioners), the School Health Service (Service Santé de la Jeunesse SSJ) and a specially established temporary vaccination structure at the Geneva University Hospital (HUG). Vaccines were made available to the physicians through the State Medical Office.

The objective of the program was to make three doses of the vaccine available as easily as possible to 11-19 years female residents of the state of Geneva in order to reach the highest possible coverage.

Target population: The target population to be vaccinated was defined by the state authorities as the 11 to 19 years old girls living in the canton of Geneva. At the beginning of the program in September 2007 approximately 24000 girls were potentially eligible, with a new cohort each year of approximately 2400 girls (data obtained from the state Population Registry).

Epidemiological monitoring: Each service provider transmitted quarterly to the State Medical Office the anonymous list of the persons who had received their first, second or third shots. The statistical analysis of the data collected was made in a way totally anonymous. The data were processed through the Téléform (c) software and analyzed to assess the HPV coverage of the target population. The data collected before December 31st 2009 was included into the study. Descriptive statistical methods with the Stata 10 software [11] were used. Confidence intervals and inferential statistics were not calculated because the study population was considered exhaustive.

\section{Results}

The global coverage rates as of December 31st 2009 (1988-1998 born cohorts) were $48.1 \%$ for one dose, $43.7 \%$ for two doses and $41.6 \%$ for three doses of the
HPV vaccine. This rates were better for the girls who were eligible at the beginning of the program have a cover age rate of $52.8 \%$ for one dose, $47.9 \%$ for two doses and $45.6 \%$ for three doses These rates were quite different from one cohort to another. For girls born in 1994 or 1995 (14 to 15 year old ones), cover rates were $68.2 \%$ and $70 \%$, respectively for the first dose, $65.6 \%$ and $68.1 \%$ for second dose and $61.9 \%$ and $65.1 \%$ for the third dose (Table 1).

The contribution to the vaccination program of the three service providers varied according to the age of the target population (Figure 1). Service providers covered different age groups. School health service (SSJ) inoculated $70 \%$ of the 14 year-old ones, $53 \%$ of the 13 yearold ones, and less than $10 \%$ of the 16 to 21 year-old ones. Medical doctors in private practice inoculated 50 to $60 \%$ of the target population except in the 13-14 year-old age group. The HUG (ad hoc vaccination center) inoculated around 50\% of the older ones (age group 19-21 years) and a much lower proportion (5\% - 15\%) of the younger ones (age group 11-14 years).

The proportion of eligible girls who did not attend the second and/or 3rd dose after receiving the first one was similar for all age groups regardless of the service providers, with the exception of the SSJ.

\section{Discussion}

Adherence is crucial for the effectiveness of a public health program. Effectiveness of a immunization program depends on high coverage and strong compliance [12]. The results of our study show that the girls who were eligible at the beginning of the program have a cover age rate of $52.8 \%$ for one dose, $47.9 \%$ for two doses and $45.6 \%$ for three doses. The cover rates for the specific target population as recommended by the Federal Committee for vaccination, i.e., the 11-14 year old ones, was $66.7 \%$ for the first, $62.7 \%$ for the second and $59.3 \%$ for the third dose.

The lower rates of vaccination for the older cohorts (1988 and 1989) might be explained by several elements: they were not the primary target group, thus less targeted by HPV vaccine promotion campaigns; they had limited access to free vaccination: indeed vaccination was free of charge only up to the 20iest birthday and they may not have yet a gynecologist and no longer a pediatrician.

The vaccination patterns according to the service provider show important variations that are most likely due to the organizational of the program as well as "consumer” habits: The School Health Service has inoculated a majority 7th graders (12-13 year old ones) because this is the main target population. The SSJ had introduced vaccination as early as the school year 2007-2008, thus fechnically before the official launch of the vaccination 
Table 1. Percentage of vaccinated girls born between 1988 and 1998 on december 31st 2009.

\begin{tabular}{|c|c|c|c|c|c|c|}
\hline Birth cohorts & $\begin{array}{c}\text { Age (years) on } \\
\text { December 31st } 2009\end{array}$ & $\begin{array}{c}\text { Number of } \\
\text { eligible girls (n) }\end{array}$ & $\begin{array}{l}\text { Vaccinated girls } \\
\text { first dose (\%) }\end{array}$ & $\begin{array}{l}\text { Vaccinated girls } \\
\text { second dose (\%) }\end{array}$ & $\begin{array}{l}\text { Vaccinated girls } \\
\text { third dose (\%) }\end{array}$ & \\
\hline 1988 & 21 & 2320 & $25.4 \%$ & $24.8 \%$ & $24.3 \%$ & \multirow{10}{*}{$\begin{array}{l}\text { Girls eligible } \\
\text { on September } \\
\text { 1st } 2008\end{array}$} \\
\hline 1989 & 20 & 2282 & $38.6 \%$ & $37.0 \%$ & $35.0 \%$ & \\
\hline 1990 & 19 & 2421 & $42.1 \%$ & $40.3 \%$ & $38.3 \%$ & \\
\hline 1991 & 18 & 2342 & $53.0 \%$ & $51.0 \%$ & $50.2 \%$ & \\
\hline 1992 & 17 & 2256 & $58.1 \%$ & $55.9 \%$ & $54.5 \%$ & \\
\hline 1993 & 16 & 2297 & $60.6 \%$ & $58.3 \%$ & $55.9 \%$ & \\
\hline 1994 & 15 & 2228 & $68.2 \%$ & $65.6 \%$ & $61.9 \%$ & \\
\hline 1995 & 14 & 2307 & $70.0 \%$ & $68.1 \%$ & $65.1 \%$ & \\
\hline 1996 & 13 & 2218 & $68.0 \%$ & $58.2 \%$ & $54.2 \%$ & \\
\hline 1997 & 12 & 2190 & $42.9 \%$ & $19.5 \%$ & $16.1 \%$ & \\
\hline Total 1988-1997 & & 22'862 & $52.8 \%$ & $47.9 \%$ & $45.6 \%$ & \multirow{3}{*}{$\begin{array}{l}\text { Girls who recently } \\
\text { entered the } \\
\text { program }\end{array}$} \\
\hline 1998 & 11 & 2201 & $2.2 \%$ & $0.8 \%$ & $0.3 \%$ & \\
\hline Total 1988-1998 & & $25^{\prime} 062$ & $48.1 \%$ & $43.7 \%$ & $41.6 \%$ & \\
\hline
\end{tabular}

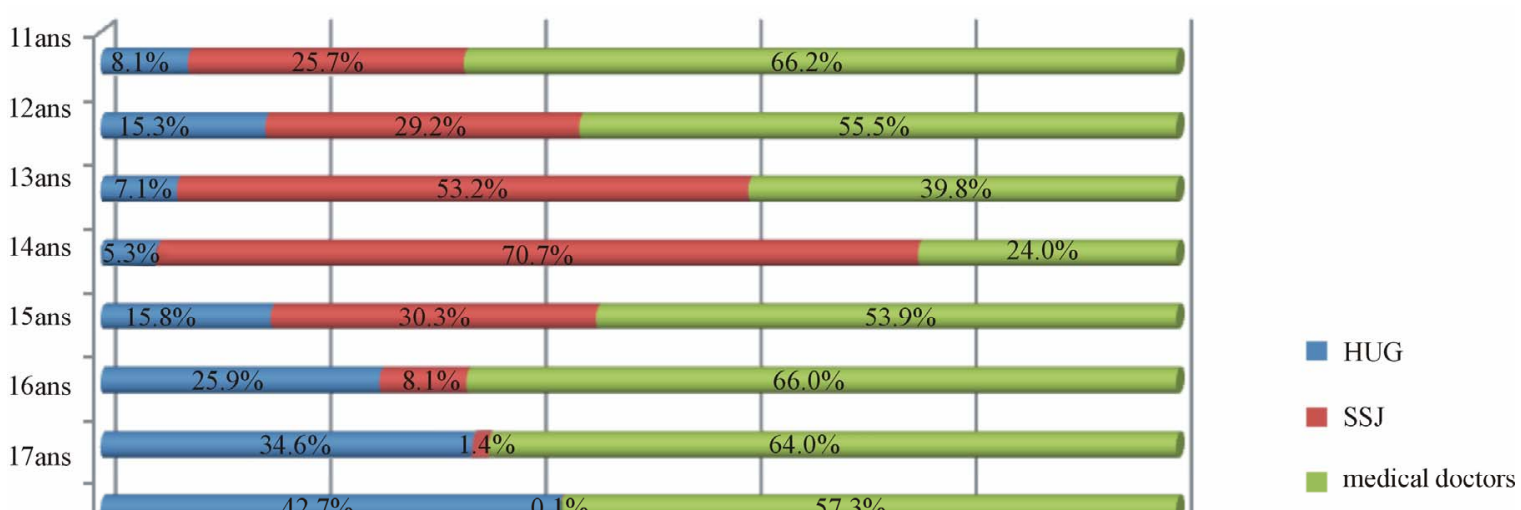

program. Medical doctors in private practice play an important role, having roughly vaccinated $50 \%$ of the target population and up to $65 \%$ in certain age groups: this might reflect the special place of practitioners have as family doctors. The HUG inoculated mostly older girls (up to roughly $50 \%$ in the $19-21$ year old group): this might reflect "an aspiration to some independence", since the HUG vaccination unit was less family-oriented than the practitioners and less school related than the SSJ.

There is only a small amount of published data on HPV vaccine coverage in areas where the vaccine was introduced. A study led in North Carolina among girls from 9 to 17 years shows cover rates of $39.7 \%$ for the 1 st dose, $34.6 \%$ for the 2nd and $25.7 \%$ for the 3rd [13]. Another study from the USA reports an HPV vaccine coverage of $44.2 \%$ for the 1 st dose, $32.3 \%$ for the 2 nd and 23.5\% for the 3rd among 13 to 17 year old girls [14]. A recent article from England reports coverage rates among 12-13 year-old girls for three doses of up to $80 \%$ [15]. From Italy coverage rates for girls born in 1996 and in 1997 of $80 \%$ for the 1 st dose, $75 \%$ for the 2nd and more than $60 \%$ for the 3rd have been reported [5].

Success of vaccination programs depends on a number of elements, including, presence of qualified health experts, availability of the vaccine, its price, the acceptabil- 
ity of the vaccine in the population, a clear promotion strategy, political support, etc [13-16]. The program initiated in Geneva by the State Medical Office has benefited from such a broad approach and gives promising results, yet the effort must be kept up. Indeed one of the key to the success of this program was the participation of the School Health Service for effective promotion of the vaccine combined with the implication of private physicians and a specially set up vaccination center. Bradin et al. have also showed the importance and the feasibility of school-based vaccine delivery [13]. Coverage data from Australia, where the vaccine policy is also based on school delivery, are very high, which calls for advocating such a strategy [17]. The possibility for the population to choose the vaccine inoculators (doctor, HUG or SSJ) seems to be one of the keys of the success of the program of immunization in the state of Geneva.

Data from Switzerland on HPV vaccination programs in the different cantons will soon be available. The Federal Office of the Public Health is in the process of launching an inventory of the HPV vaccination programs implemented in the various cantons, addressing issues such as the adopted methodology and the obtained coverage rates: this should give an idea of the HPV vaccination coverage at the country level.

\section{REFERENCES}

[1] Swiss Association of the Registers of Tumors (ASRT), “Statistics of Cancer Incidence 1986-2005,” 2008. http://www.asrt.ch

[2] National Institute for Cancer Epidemiology and Registration (NICR), 2007. http://asrt.ch/nicer/stat/i5ch8307.pdf

[3] Federal Office of Public Health, "Recommendations for Vaccination against the Human Papillomavirus (HPV)," 2008.

[4] Federal Office of Public Health, "Vaccination against HPV - The Beginning of the County Programs of Vaccination,” 2008, pp. 656-659.

[5] J. Lugarini and F. Maddalo, "Results of a Vaccination Campaign against Human Papillomavirus in the Province of La Spezia,” Euro. Surveill 2009, Liguria, Italy, MarchDecember 2008.
[6] World Health Organization, Report of the Consultation on Human Papillomavirus Vaccines, 2005.

[7] C. Simoens, M. Sabbe, D. P. Van, P. Beutels and M. Arbyn, "Introduction of Human Papillomavirus (HPV) Vaccination in Belgium,” Euro.Surveill 2009, 2007-2008.

[8] Federal Commissions for the Vaccinations, "Recommendation for the Basic Vaccination of the Teenagers against Cervical Cancer and the Other Diseases Caused by the Human Papilloma Virus (HPV),” 2007.

[9] Geneva Cancer Registry, “The Cancer in Geneva,” 2009.

[10] K. Louie, M. N. Didelot, A. Damay, N. Nagot, P. Mayaud and M. Segondy, "Papillomavirus humains (HPV) et cancers associés: aspects épidémiologiques,” Revue Francophone des Laboratoires, Vol. 2008, No. 405, 2008, pp. 27-34.

[11] StataCorp. Stata 10, 25 July 2007.

[12] R. Rouzier and J. P. Giordanella, “Coverage and Compliance of Human Papilloma Virus Vaccines in Paris: Demonstration of Low Compliance with Non-SchoolBased Approaches,” Journal of Adolescent Health, Vol. 47, No. 3, 2010, pp. 237-241. doi:10.1016/j.jadohealth.2010.04.006

[13] C. S. Berry-Caban and J. B. Buenaventura, "HPV Vaccination Coverage among Adolescents Aged 9 to 17 Years in a United States Military Treatment Facility,” International Journal of Adolescent Medicine and Health, Vol. 21, No. 4, 2009, pp. 567-570.

[14] Center of Disease and Control (CDC), "StatBite: HPV Vaccination among U. S. Girls Aged 13-17 Years in 2007,” Journal of the National Cancer Institute, Vol. 100, 2008, p. 1570. doi:10.1093/jnci/djn417

[15] K. Canfell, "Monitoring HPV Vaccination Programmes," British Medical Journal (BMJ), 2010, 340: c1666.

[16] L. Brabin, S. A. Roberts, R. Stretch, et al., "Uptake of First Two Doses of Human Papillomavirus Vaccine by Adolescent Schoolgirls in Manchester: Prospective Cohort Study,” British Medical Journal (BMJ), Vol. 336, No. 7652, 2008, pp. 1056-1058. doi:10.1136/bmj.39541.534109.BE

[17] J. M. Brotherton, S. L. Deeks, S. Campbell-Lloyd, et al., "Interim Estimates of Human Papillomavirus Vaccination Coverage in the School-Based Program in Australia," Communicable Diseases Intelligence, Vol. 32, No. 4, December 2008, pp. 457-61. 